\title{
Biharmonic Green Functions on Homogeneous Trees
}

\author{
Joel M. Cohen*, Flavia Colonna and David Singman
}

\begin{abstract}
The study of biharmonic functions under the ordinary (Euclidean) Laplace operator on the open unit disk $\mathbb{D}$ in $\mathbb{C}$ arises in connection with plate theory, and in particular, with the biharmonic Green functions which measure, subject to various boundary conditions, the deflection at one point due to a load placed at another point. A homogeneous tree $T$ is widely considered as a discrete analogue of the unit disk endowed with the Poincaré metric. The usual Laplace operator on $T$ corresponds to the hyperbolic Laplacian. In this work, we consider a bounded metric on $T$ for which $T$ is relatively compact and use it to define a flat Laplacian which plays the same role as the ordinary Laplace operator on $\mathbb{D}$. We then study the simply-supported and the clamped biharmonic Green functions with respect to both Laplacians.
\end{abstract}

Mathematics Subject Classification (2000). 31C20.

Keywords. Biharmonic, trees, harmonic.

\section{Introduction}

Let $\Delta_{E}$ be the ordinary Laplace operator in the complex plane, so that for a $C^{2}$ function $f$ on some domain in $\mathbb{C}$,

$$
\Delta_{E} f=f_{x x}+f_{y y}=4 f_{z \bar{z}} .
$$

When restricting to functions in $\mathbb{D}=\left\{(x, y) \in \mathbb{R}^{2}: x^{2}+y^{2}<1\right\}$ (which we identify with $\{z \in \mathbb{C}:|z|<1\}$ ), this operator can be viewed as the Laplace-Beltrami operator with respect to the Euclidean metric $d s_{E}^{2}=d x^{2}+d y^{2}$. On $\mathbb{D}$ there is also the Laplace-Beltrami operator $\Delta_{H}$ relative to the hyperbolic (Poincaré) metric

$$
d s_{H}^{2}=\left(1-x^{2}-y^{2}\right)^{-2}\left(d x^{2}+d y^{2}\right)=\left(1-|z|^{2}\right)^{-2} d s_{E}^{2},
$$

\footnotetext{
${ }^{*}$ Corresponding author.
} 
which is invariant under the action of the biholomorphic maps (see [22, Chapter 4]). The relation between $\Delta_{E}$ and $\Delta_{H}$ is

$$
\Delta_{H} f(z)=\left(1-|z|^{2}\right)^{2} \Delta_{E} f(z), f \in C^{2}(\mathbb{D}), z \in \mathbb{D} .
$$

A harmonic function $f$ is a solution of $\Delta_{E} f=0$, or equivalently, $\Delta_{H} f=$ 0. A Euclidean (respectively, hyperbolic) biharmonic function $f$ is a solution of $\Delta_{E}^{2} f=0$ (respectively, $\Delta_{H}^{2} f=0$ ). While the sets of hyperbolic and Euclidean harmonic functions are the same, the analogous two sets of biharmonic functions are different.

The Euclidean biharmonic functions arise in connection with various physical problems, such as the deflection of a thin plate, elasticity, and radar imaging (see [4] and [20]). For applications in potential theory and complex analysis see [1],[2],[3] and [14].

Corresponding to each of the above two Laplacians on $\mathbb{D}$, we can define the biharmonic Green functions $\Gamma_{S}(z, w)$ and $\Gamma_{C}(z, w)$ called, respectively, the simplysupported and clamped biharmonic Green function. They both have the property that the bilaplacian applied in the $z$-variable gives a point mass at $w$, and both vanish as $z$ tends to any point of the unit circle. The function $\Gamma_{S}$ has the additional property that the Laplacian applied to it in the first variable tends to 0 at each point of the unit circle, whereas the normal derivative of $\Gamma_{C}$ in the first variable vanishes on the unit circle. Here, the normal derivative is defined with respect to the Riemannian metric [23, page 16]. All four of these biharmonic Green functions exist and are unique (see [23]). In the case of the Euclidean metric, if $\mathbb{D}$ represents a thin plate, then both biharmonic Green functions at $(z, w)$ represent the resulting deflection at $z$ due to a unit load at $w$. In both cases the edge of the plate remains fixed. In the clamped case the plate is horizontally welded at the edge, while in the simply-supported case the edge of the plate is merely resting on a support so that the slope there can change.

Discretizations of many classical problems in harmonic analysis, potential theory, geometry, functional analysis, and differential equations on fractals have been considered. Some developments include, among many other works, [5],[6],[7], [8],[9],[10],[11],[12],[13],[15],[16],[17],[18],[19],[24].

In this paper we consider on a homogeneous tree analogues of the simplysupported and clamped biharmonic Green functions. A homogeneous tree under the distance that counts the number of edges between two vertices is widely regarded as a discrete analogue of the hyperbolic disk in the complex plane. The resulting Laplacian (which is the one most commonly used) defined as the averaging operator minus the identity is invariant under the action of the isometries on the tree. Hence, we call this invariant Laplacian the hyperbolic Laplacian. Both hyperbolic biharmonic Green functions on a tree are straightforward to calculate (Corollary 1.1).

Most of the paper is dedicated to the more difficult task of studying the analogues of the Euclidean biharmonic Green functions on the tree. For this purpose 
we work with a bounded metric that makes the tree relatively compact. This metric, defined in this section (see (1.5)), has been considered before, and we shall refer to it as a Euclidean or flat metric. We use it to define the normal derivative at a boundary point of a function on the tree and, motivated by (1.2), we define a Laplace operator which we call the flat Laplacian. Corresponding to the flat Laplacian we are able to write the simply-supported biharmonic Green function $\Gamma_{S}(u, v)$ in closed form (see Theorem 2.2). Using $\Gamma_{S}$ we prove the existence and uniqueness of the clamped biharmonic Green function $\Gamma_{C}(u, v)$ (see Theorem 5.1 and Theorem 6.3), but we have been unable to find an explicit formula for it.

Many of the calculations in this paper were carried out with the help of the computer software Maple ${ }^{1}$.

\subsection{Preliminary definitions on trees}

A tree is a locally finite connected graph with no loops, which, as a set, we identify with the collection of its vertices. Two vertices $v$ and $w$ of a tree are called neighbors if there is an edge connecting them, in which case we use the notation $v \sim w$. A vertex is called terminal if it has exactly one neighbor. A path is a finite or infinite sequence of neighboring vertices which are all distinct. An infinite path starting at a vertex is called a ray. For any two vertices $u$ and $v$, let $[u, v]$ denote the unique path joining $u$ and $v$ and let $|u-v|$ be the number of edges in the path $[u, v]$.

Fixing a vertex $e \in T$ as a root of the tree, the predecessor $u^{-}$of a vertex $u$, with $u \neq e$, is the next to the last vertex of the path from $e$ to $u$. We say that $v$ is an ancestor of $u$ and $u$ is a descendant of $v$, if $v$ is in the path from $e$ to $u^{-}$. The sector $S_{v}$ is the set consisting of $v$ and all its descendants. We call children of a vertex $v$ the vertices $u$ such that $u^{-}=v$. For brevity we use the notation $|u|$ for $|u-e|$.

The boundary $\partial T$ of $T$ is the union of the set of equivalence classes of rays under the equivalence relation generated by the unit shift together with the terminal vertices. For any vertex $u$, we denote by $[u, \omega)$ the (unique) path starting at $u$ in the class $\omega$; then $\partial T$ can be identified with the set of paths starting at $u$. Furthermore, $\partial T$ is a compact space under the topology generated by the sets

$$
I_{v}=\{\omega \in \partial T: v \in[e, \omega)\},
$$

which we call intervals. Clearly, $\Omega=I_{E}$. For $v \in T, n \in \mathbb{N}$, with $n \leq|v|$, define $v_{n}$ to be the vertex of length $n$ in the path $[e, v]$. Similarly, for a class $\omega$, the path $[e, \omega)$ will be denoted by $\left[\omega_{0}, \omega_{1}, \omega_{2}, \ldots\right]$. If $v$ and $w$ are vertices or boundary points, define $v \wedge w$ to be the vertex $v_{k}$ such that $v_{k}=w_{k}$ and $v_{k+1} \neq w_{k+1}$.

Observe that if $\left[v_{0}, v_{1}, \ldots\right]$ is an infinite path starting at $e$, then $I_{v_{j}}-I_{v_{j+1}}$ can be written as the disjoint union

$$
I_{v_{j}}-I_{v_{j+1}}=\bigcup_{u \in C_{j}} I_{u},
$$

\footnotetext{
${ }^{1}$ Maple is a registered trademark of Waterloo Maple Inc.
} 
where $C_{j}$ is the set consisting of the children of $v_{j}$ unequal to $v_{j+1}$. Thus, for each $v \in T$ with $|v|=n$,

$$
\Omega=\bigcup_{j=0}^{n-1}\left(I_{v_{j}}-I_{v_{j+1}}\right) \cup I_{v},
$$

which by (1.3) can be written as a finite disjoint union of intervals.

By a homogeneous tree of degree $q+1$ (with $q \geq 2$ ) we mean a tree all of whose vertices have exactly $q+1$ neighbors.

From this point on we shall let $T$ be a homogeneous tree of degree $q+1$ rooted at vertex $e$.

By the Lebesgue measure on $\partial T$ we mean the unique probability measure whose value on $I_{v}$ is $\frac{q}{q+1} q^{-|v|}$ for $v \neq e$.

The hyperbolic metric on $T$ is the metric whose associated distance between two vertices $u$ and $v$ is $|u-v|$. The automorphisms of the tree are isometries with respect to the hyperbolic metric on $T$ in analogy with the Möbius transformations of $\mathbb{D}$ which are isometries of the hyperbolic disk.

The flat metric we consider is the metric $d$ on $T$ such that

$$
d(v, \partial T)=q^{-|v|} .
$$

Therefore the length of an edge $\left[v^{-}, v\right]$ is given by

$$
q^{-(|v|-1)}-q^{-|v|}=(q-1) q^{-|v|} .
$$

In analogy to the Euclidean distance between 0 and $\partial \mathbb{D}$, the distance between $e$ and $\partial T$ is 1 . Furthermore, the only isometries of the flat tree are the tree automorphisms which fix e, in analogy with the fact that the only Möbius transformations which preserve the Euclidean distance are those that fix 0 (i.e. the rotations about the origin).

As is customary, by a function on a tree $T$ we mean a function on its set of vertices. For a function $f$ on $T$ the hyperbolic Laplacian of $f$, which is the most commonly used Laplacian, is defined by

$$
\Delta_{H} f(v)=\frac{1}{q+1} \sum_{w \sim v} f(w)-f(v),
$$

where $w \sim v$ means that $w$ and $v$ are neighbors, i.e. they are connected by an edge. The flat Laplacian of $f$ is defined as

$$
\Delta_{E} f(v)=q^{2|v|} \Delta_{H} f(v), v \in T .
$$

This is motivated by (1.2) because $1-|z|^{2}$ is essentially the distance from $z$ to the boundary in the unit disk and $q^{-|v|}$ is the distance from $v$ to $\partial T$.

Clearly, as in the case of the unit disk, the harmonic functions with respect to the operator $\Delta_{E}$ are the same as the harmonic functions with respect to $\Delta_{H}$. However, the two classes of biharmonic functions $f$ satisfying $\Delta_{E}^{2} f=0$ and $\Delta_{H}^{2} f=$ 0 are quite different. 
For $u, v \in T$, let $G_{H}(u, v)=\frac{q}{q-1} q^{-|u-v|}$ denote the hyperbolic Green function and for $\omega \in \partial T$, let

$$
K_{H}(u, \omega)=q^{2|u \wedge \omega|-|u|}
$$

be the hyperbolic Martin kernel. A hyperbolic Green potential is a function of the form

$$
G_{H} f(u)=\sum_{w \in T} G_{H}(u, w) f(w),
$$

where $f$ is a function on $T$ for which

$$
\sum_{w \in T} G_{H}(e, w)|f(w)|<\infty .
$$

Then $G_{H} f$ is finite-valued on $T$ and satisfies the Poisson equation

$$
\Delta_{H}\left(G_{H} f\right)=-f .
$$

For a finite Borel measure $\mu$ on $T$, the hyperbolic Martin integral of $\mu$ is given by

$$
K_{H} \mu(u)=\int K_{H}(u, \omega) d \mu(\omega)=\int q^{2|u \wedge \omega|-|u|} d \mu(\omega) .
$$

The above formulas are developed fully in [6].

Observe that there is a flat harmonic Green function which is given by

$$
G_{E}(u, w)=\frac{q}{q-1} q^{-|u-w|-2|w|},
$$

as one can immediately verify by showing that its flat Laplacian with respect to $u$ is $-\delta_{w}$. However, there is no nearest-neighbor transition probability that produces $\Delta_{E}$. Indeed, if we assumed the existence of such a transition probability $p$ and

$$
\Delta_{E} f(u)=\sum_{w \sim v} p(u, w) f(w)-f(u)
$$

then for $f=\delta_{v}$ for some vertex $v \neq e$ we would obtain $\Delta_{E} f(v)=-1$. But $\Delta_{H} f(v)$ is also -1 , contradicting the definition $\Delta_{E} f(v)=q^{2|v|} \Delta_{H} f(v)$.

For a function $f$ on $T$ we define various kinds of normal derivatives. For $v \neq e$ we define the hyperbolic normal derivative of $f$ at $v$ by

$$
\frac{\partial f}{\partial n}(v)=f(v)-f\left(v^{-}\right)
$$

For $\omega$ a boundary point of $T$, we define the hyperbolic normal derivative of $f$ at $\omega$ by

$$
\frac{\partial f}{\partial n}(\omega)=\lim _{m \rightarrow \infty} \frac{\partial f}{\partial n}\left(\omega_{m}\right)
$$

and if we can extend $f$ to $\omega$ by the formula

$$
f(\omega)=\lim _{m \rightarrow \infty} f\left(\omega_{m}\right)
$$

then we define the flat normal derivative of $f$ at $\omega$ by

$$
\partial_{n} f(\omega)=\lim _{m \rightarrow \infty} \frac{f\left(\omega_{m}\right)-f(\omega)}{q^{-m}},
$$


provided these limits exist.

The simply-supported flat biharmonic Green function $\Gamma_{S}$ and the clamped flat biharmonic Green function $\Gamma_{C}$ are respectively real-valued continuous functions defined on $\bar{T} \times T$ such that for each $v \in T$,

$$
\begin{cases}\left(\Delta_{E}\right)_{u}^{2} \Gamma_{S}(u, v)=\delta_{v}(u) & \text { for all } u \in T \\ \Gamma_{S}(\omega, v)=0 & \text { for all } \omega \in \partial T \\ \lim _{u \rightarrow \omega}\left(\Delta_{E}\right)_{u} \Gamma_{S}(u, v)=0 & \text { for all } \omega \in \partial T\end{cases}
$$

and

$$
\begin{cases}\left(\Delta_{E}\right)_{u}^{2} \Gamma_{C}(u, v)=\delta_{v}(u) & \text { for all } u \in T \\ \Gamma_{C}(\omega, v)=0 & \text { for all } \omega \in \partial T \\ \partial_{n} \Gamma_{C}(\omega, v)=0 & \text { for all } \omega \in \partial T\end{cases}
$$

where the subscript refers to the variable used in the calculation of the Laplacian and $\delta_{v}$ is the Kronecker delta function.

The existence and uniqueness of $\Gamma_{S}$ are proved in Theorems 2.1 and 2.2. The corresponding theorems for $\Gamma_{C}$ are harder to prove. The existence is shown in Theorem 5.1 and the uniqueness is shown in Theorem 6.3. The proof of the existence of $\Gamma_{C}$ requires the use of $\Gamma_{S}$. In a Riemannian manifold setting, the same is true [21].

We define the hyperbolic simply-supported and clamped biharmonic Green functions as above with $\Delta_{E}$ replaced by $\Delta_{H}$ and $\partial_{n} \Gamma_{C}(\omega, v)$ replaced by $\frac{\partial \Gamma_{C}}{\partial n}(\omega, v)$. Because of the symmetry of the tree and the translation invariance of the hyperbolic Laplacian, it seems intuitively obvious that these biharmonic Green functions are unique. The formal proof of the uniqueness is similar to the proofs on the flat tree given in Theorem 2.1 and Theorem 6.3 so we omit them. The hyperbolic biharmonic Green functions are much easier to deal with than $\Gamma_{S}$ and $\Gamma_{C}$ and, in fact, are equal. We give the formula in Corollary 1.1.

In the following proposition, a function defined on a sector $S_{v}$ is called radial if its value at each vertex $u \in S_{v}$ depends only on $|u-v|$.

Proposition 1.1. The radial functions defined on a sector $S_{v}$ that are flat (respectively, hyperbolic) biharmonic at every vertex $u$ in $S_{v}$ such that $|u-v|>1$ are precisely the linear combinations of $1, q^{-|u-v|}, q^{-2|u-v|}$ and $q^{-3|u-v|}$ (respectively, $1,|u-v|, q^{-|u-v|}$ and $\left.|u-v| q^{-|u-v|}\right)$.

Proof. Since $|u-v|=|u|-|v|$ on $S_{v}$, it suffices for us to prove the result in case $v=e$. Denote by $f_{n}$ the value of the radial function $f(u)$ where $|u|=n$. Then $\Delta_{E} f$ and $\Delta_{H} f$ are also radial. The equation $\Delta_{E}^{2} f=0$ has the same solutions as 
$\Delta_{H} \Delta_{E} f=0$, which can be written as

$$
\begin{aligned}
0= & \frac{q}{q+1}\left(\Delta_{E} f\right)_{n+1}+\frac{1}{q+1}\left(\Delta_{E} f\right)_{n-1}-\left(\Delta_{E} f\right)_{n} \\
= & \frac{q}{q+1}\left[\frac{q}{q+1} f_{n+2}+\frac{1}{q+1} f_{n}-f_{n+1}\right] q^{2(n+1)} \\
& +\frac{1}{q+1}\left[\frac{q}{q+1} f_{n}+\frac{1}{q+1} f_{n-2}-f_{n-1}\right] q^{2(n-1)} \\
& -\left[\frac{q}{q+1} f_{n+1}+\frac{1}{q+1} f_{n-1}-f_{n}\right] q^{2 n},
\end{aligned}
$$

and the equation $\Delta_{H}^{2} f=0$ is the same with the factors $q^{2(n+1)}, q^{2(n-1)}, q^{2 n}$ removed. Multiplying by $(q+1)^{2}$ and dividing by $q^{2(n-1)}$, equation $(1.14)$ reduces to a fourth order, linear, homogeneous difference equation. The corresponding characteristic equation is

$$
q^{6} r^{4}-q^{3}(q+1)\left(q^{2}+1\right) r^{3}+q\left(q^{2}+1\right)\left(q^{2}+q+1\right) r^{2}-(q+1)\left(q^{2}+1\right) r+1=0,
$$

whose roots are $1,1 / q, 1 / q^{2}, 1 / q^{3}$.

Similarly, the equation $\Delta_{H}^{2} f=0$ yields a fourth order, linear, homogeneous difference equation whose associated characteristic equation is

$$
q^{2} r^{4}-2 q(q+1) r^{3}+\left(q^{2}+4 q+1\right) r^{2}-2(q+1) r+1=0 .
$$

Its roots are 1 and $1 / q$, each with multiplicity 2 .

Using Proposition 1.1 we now determine both the hyperbolic simply-supported and clamped biharmonic Green functions on $T$.

Corollary 1.1. The hyperbolic simply-supported and clamped biharmonic Green functions are equal to each other and are given by

$$
\Gamma(u, v)=\left[\frac{q^{2}+1+\left(q^{2}-1\right)|u-v|}{(q-1)^{3}}\right] q^{-(|u-v|-1)} .
$$

Proof. By the proposition, the radial functions on $T$ that tend to 0 at $\partial T$ and which are hyperbolic biharmonic at all vertices $u$ with $|u|>1$ are of the form $f(u)=(a+b|u|) q^{-|u|}$ for constants $a$ and $b$. A simple calculation shows that for each boundary point $\omega$ and any choice of $a$ and $b, \Delta_{H} f(u) \rightarrow 0$ as $u \rightarrow \omega$ and $\frac{\partial f}{\partial n}(\omega)=0$. The additional restrictions that $\Delta_{H}^{2} f(u)=0$ for all $|u|=1$ and $\Delta_{H}^{2} f(e)=0$ lead to a set of two linear equations in the unknowns $a$ and $b$ for which the solution is $a=q\left(q^{2}+1\right) /(q-1)^{3}$ and $b=q\left(q^{2}-1\right) /(q-1)^{3}$. This then gives us a single function $\Gamma(u, e)$ that satisfies the defining properties of the hyperbolic simply-supported and clamped biharmonic Green functions. The formula for $\Gamma(u, v)$ follows by translation.

Remark 1.1. Of the radial flat biharmonic functions in Proposition 1.1, only the last three go to 0 at infinity. Of these last three, only the second and fourth have a flat Laplacian that is zero at infinity and only the last two have a flat normal derivative at infinity that is zero. Thus, we expect the flat simply-supported 
biharmonic Green function, $\Gamma_{S}$, with respect to $e$ be written as a linear combination of $q^{-|u|}$ and $q^{-3|u|}$, and with respect to a fixed vertex $v$, as a linear combination of $q^{-|u-u \wedge v|}$ and $q^{-3|u-u \wedge v|}$ on the set of vertices $u$ such that $u \wedge v=v_{j}$, for some $j=0, \ldots,|v|$. Similarly, we expect the flat clamped biharmonic Green function, $\Gamma_{C}$, to be written as a linear combination of $q^{-2|u-u \wedge v|}$ and $q^{-3|u-u \wedge v|}$ on that same set. The coefficients in these linear combinations depend only on $|u \wedge v|$ and $|v|$.

Definition 1.1. A function of two variables $u$ and $v$ on $T$ is biradial if it only depends on $|u|,|v|$, and $|u-v|$ or, equivalently, on $|u|,|v|$, and $|u \wedge v|$, since $|u|+|v|=$ $|u-v|+2|u \wedge v|$.

Thus, biradial functions are invariant under all automorphisms that fix $e$. We shall prove that both biharmonic Green functions $\Gamma_{S}$ and $\Gamma_{C}$ are biradial.

\section{Flat Simply-supported biharmonic Green function}

We first deal with the question of uniqueness.

Theorem 2.1. The flat simply-supported biharmonic Green function is unique.

Proof. The difference of two such Green functions gives in the $u$ variable a function $f(u)$ that is flat biharmonic on $T$ with the property that $f(u)$ and $\Delta_{E} f(u)$ both tend to 0 at every boundary point of $T$. It is easily proved using the Maximum Principle that a function harmonic on $T$ which tends to 0 at every boundary point of $T$ is necessarily identically 0 . The result then follows by applying this fact first to the harmonic function $\Delta_{E} f$ and then to $f$.

In the following theorem we give two formulas for the flat simply-supported biharmonic Green function.

Theorem 2.2. The flat simply-supported biharmonic Green function is given by

$$
\Gamma_{S}(u, v)=\left(\frac{q}{q-1}\right)^{2} q^{-2|v|} \sum_{w \in T} q^{-2|w|} q^{-|u-w|} q^{-|v-w|},
$$

and also by

$$
\Gamma_{S}(u, v)=\frac{x_{S}(u \wedge v, v)}{q^{|u-u \wedge v|}}+\frac{y_{S}(u \wedge v, v)}{q^{3|u-u \wedge v|}},
$$

where

$$
x_{S}(u \wedge v, v)=\frac{q\left(q^{-3|v|-|u \wedge v|}\left[\left(q^{2}+1\right)^{2}+|u \wedge v|\left(q^{4}-1\right)\right]-q^{2} q^{-5|v|+|u \wedge v|}\right)}{(q-1)^{2}\left(q^{3}-1\right)}
$$

and

$$
y_{S}(u \wedge v, v)=-\frac{q^{3}}{(q-1)^{2}\left(q^{3}-1\right)} q^{-3|v|-|u \wedge v|} .
$$


Proof. Let $v$ be a fixed vertex throughout this proof. Since $q^{-2|w|}$ is summable over $T$, it follows from the Dominated Convergence Theorem that the right-hand side of (2.1) goes to 0 as $u$ goes to the boundary. Furthermore, using (1.7) the application of $\Delta_{E}$ to the right-hand side in the $u$ variable yields $-q /(q-1) q^{-2|v|} q^{-|u-v|}$, which also goes to 0 as $u$ goes to the boundary, while $\Delta_{E}^{2}$ applied to the right-hand side yields the characteristic function of $v$. Then (2.1) follows from this, (1.12), and Theorem 2.1.

To prove (2.2), consider $\{u \in T:|u \wedge v|=m\}$ for each $m$ from 0 to $|v|$. It follows from (2.1) that $u \mapsto \Gamma_{S}(u, v)$ is radial on this set. Thus (2.2) follows from Proposition 1.1 and Remark 1.1, though the functions $x_{S}$ and $y_{S}$ are still to be determined.

We show below that we are able to sum the expression in (2.1) in closed form in the special case that $u=u \wedge v$ (i.e. when $u$ lies on the geodesic $[e, v]$ ) and also in the special case that $u$ is not a point on this geodesic but is a neighbor of such a point. Let $\Gamma_{1}$ denote $\Gamma_{S}(u, v)$ in this first case, and let $\Gamma_{2}$ denote $\Gamma_{S}(u, v)$ in the second case. Plugging these into (2.2) yields

$$
\begin{aligned}
& \Gamma_{1}=x_{S}(u \wedge v, v)+y_{S}(u \wedge v, v), \\
& \Gamma_{2}=\frac{x_{S}(u \wedge v, v)}{q}+\frac{y_{S}(u \wedge v, v)}{q^{3}}
\end{aligned}
$$

from which we deduce

$$
x_{S}(u \wedge v, v)=\frac{q^{3} \Gamma_{2}-\Gamma_{1}}{q^{2}-1}, \quad y_{S}(u \wedge v, v)=\frac{q^{2}\left(\Gamma_{1}-q \Gamma_{2}\right)}{q^{2}-1} .
$$

We now calculate $\Gamma_{1}$ and $\Gamma_{2}$. Let us denote $|v|$ by $n$ and $|u \wedge v|$ by $m$. A simple combinatorial argument gives $\Gamma_{1}$ and $\Gamma_{2}$ in the following non-closed form:

$$
\begin{aligned}
\Gamma_{1}= & \left(\frac{q}{q-1}\right)^{2} q^{-2 n}\left[\sum_{k=0}^{\infty} q^{-2 k} q^{-(n+k)} q^{-(m+k)} q^{k}+\sum_{k=0}^{\infty} q^{-2(n+k)} q^{-k} q^{-(n-m+k)} q^{k}\right. \\
& +\sum_{j=1}^{m}\left(q^{-2 j} q^{-(n-j)} q^{-(m-j)}+\sum_{k=1}^{\infty} q^{-2(j+k)} q^{-(n-j+k)} q^{-(m-j+k)}(q-1) q^{k-1}\right) \\
& \left.+\sum_{j=m+1}^{n-1}\left(q^{-2 j} q^{-(n-j)} q^{-(j-m)}+\sum_{k=1}^{\infty} q^{-2(j+k)} q^{-(n-j+k)} q^{-(j-m+k)}(q-1) q^{k-1}\right)\right]
\end{aligned}
$$

and 


$$
\begin{aligned}
\Gamma_{2}= & \left(\frac{q}{q-1}\right)^{2} q^{-2 n}\left[\sum_{k=0}^{\infty} q^{-2 k} q^{-(n+k)} q^{-(m+k+1)} q^{k}+\sum_{k=0}^{\infty} q^{-2(n+k)} q^{-k} q^{-(n-m+k+1)} q^{k}\right. \\
& +\sum_{j=1}^{m-1}\left(q^{-2 j} q^{-(n-j)} q^{-(m+1-j)}+\sum_{k=1}^{\infty} q^{-2(j+k)} q^{-(n-j+k)} q^{-(m-j+k+1)}(q-1) q^{k-1}\right) \\
& +\sum_{j=m+1}^{n-1}\left(q^{-2 j} q^{-(n-j)} q^{-(j-m+1)}+\sum_{k=1}^{\infty} q^{-2(j+k)} q^{-(n-j+k)} q^{-(j-m+k+1)}(q-1) q^{k-1}\right) \\
& +q^{-2 m} q^{-(n-m)} q^{-1}+\sum_{k=0}^{\infty} q^{-2(m+1+k)} q^{-(n-m+1+k)} q^{-k} q^{k} \\
& \left.+\sum_{k=1}^{\infty} q^{-2(m+k)} q^{-(n-m+k)} q^{-(k+1)}(q-2) q^{k-1}\right] .
\end{aligned}
$$

A calculation gives

$$
\Gamma_{1}=\frac{q}{(q-1)^{2}\left(q^{3}-1\right)}\left[q^{-3 n-m}\left(q^{4}+q^{2}+1+m\left(q^{4}-1\right)\right)-q^{-5 n+m} q^{2}\right]
$$

and

$$
\Gamma_{2}=\frac{1}{(q-1)^{2}\left(q^{3}-1\right)}\left[q^{-3 n-m}\left(q^{4}+2 q^{2}+m\left(q^{4}-1\right)\right)-q^{-5 n+m} q^{2}\right] .
$$

Plugging these into (2.5) and replacing $n$ by $|v|$ and $m$ by $|u \wedge v|$ gives (2.3) and (2.4).

\section{Flat Biharmonic Martin kernel}

We introduce the following function which will be useful for constructing examples of functions that are flat biharmonic on $T$.

Definition 3.1. The (flat) biharmonic Martin kernel is defined on $T \times \partial T$ by

$$
B(u, \omega)=\frac{q}{q-1} \sum_{w \in T} \frac{K_{H}(w, \omega)}{q^{|u-w|} q^{2|w|}}=\frac{q}{q-1} \sum_{w \in T} \frac{q^{2|w \wedge \omega|-|w|}}{q^{|u-w|} q^{2|w|}} .
$$

If $\mu$ is a finite Borel measure on $\partial T$, then the (flat) biharmonic Martin integral of $\mu, B \mu(u)$, is given by

$$
B \mu(u)=\int_{\partial T} B(u, \omega) d \mu(\omega)
$$

Theorem 3.1. Let $\mu$ be a finite Borel measure on $\partial T$ such that $q^{-|u|} K_{H} \mu(u)$ is bounded on $T$. Then $B \mu$ is a hyperbolic Green potential that is flat biharmonic on $T$, and satisfies

$$
\Delta_{H}(B \mu)(u)=-\frac{K_{H} \mu(u)}{q^{2|u|}} \text { and } \Delta_{E}(B \mu)(u)=-K_{H} \mu(u) .
$$

In particular

$$
\Delta_{H}(B(u, \omega))=-\frac{q^{2|u \wedge \omega|}}{q^{3|u|}}
$$


Furthermore

$$
\lim _{u \rightarrow \omega} B \mu(u)=0 \text { for every } \omega \in \partial T .
$$

Proof. By Fubini's Theorem the formula for $B \mu$ can also be written as

$$
B \mu(u)=\frac{q}{q-1} \sum_{w \in T} \frac{K_{H} \mu(w)}{q^{|u-w|} q^{2|w|}} .
$$

Since $q^{-2|w|}$ is summable over $T$, it follows that $B \mu(u)$ is a Green potential in case $K_{H} \mu(w)$ is bounded by a multiple of $q^{|w|}$. The formulas in (3.2) then follow from (1.7). Formula (3.3) follows as a special case since $B(u, \omega)$ can be obtained from $B \mu(u)$ by taking $\mu$ to be the point mass at $\omega$.

Since $K_{H} \mu(w)$ is bounded above by a constant multiple of $q^{|w|}$, it follows from (3.5) that $B \mu(u)$ is bounded above by a constant multiple of $\sum_{w \in T} q^{-|w-u|} q^{-|w|}$, so in order to prove (3.4) it suffices to show that this sum goes to 0 as $|u|$ goes to $\infty$. Denoting $n$ by $|u|, m$ by $|u \wedge w|$ and $k$ by $|w-w \wedge u|$ we see that the sum is given by

$$
\begin{aligned}
\sum_{k=0}^{\infty} \frac{1}{q^{n+k} q^{k}} q^{k} & +\sum_{m=1}^{n}\left[\frac{1}{q^{n-m} q^{m}}+\sum_{k=1}^{\infty} \frac{1}{q^{n-m+k} q^{m+k}}(q-1) q^{k-1}\right] \\
& +\sum_{m=n+1}^{\infty}\left[\frac{1}{q^{m-n} q^{m}}+\sum_{k=1}^{\infty} \frac{1}{q^{m-n+k} q^{m+k}}(q-1) q^{k-1}\right] .
\end{aligned}
$$

An analysis of each term in this sum shows that it is dominated by a multiple of $n q^{-n}$. This is confirmed by the fact that this sum equals $q^{-n}\left(\frac{n\left(q^{2}-1\right)+q^{2}+1}{q(q-1)}\right)$. Thus, letting $n \rightarrow \infty$ we obtain (3.4).

In the next theorem we obtain a formula for $B(u, \omega)$ in closed form.

Theorem 3.2. For $u \in T, \omega \in \partial T$, we have

$$
B(u, \omega)=\frac{q^{-|u|}}{(q-1)\left(q^{3}-1\right)}\left[\left(q^{2}+1\right)^{2}+\left(q^{4}-1\right)|u \wedge \omega|-q^{2} q^{-2|u-u \wedge \omega|}\right] .
$$

If $\mu$ is a finite Borel measure on $\partial T$, then

$$
B \mu(u)=\int_{\partial T} \frac{q^{-|u|}}{(q-1)\left(q^{3}-1\right)} \cdot\left[\left(q^{2}+1\right)^{2}+\left(q^{4}-1\right)|u \wedge \omega|-q^{2} q^{-2|u-u \wedge \omega|}\right] d \mu(\omega) .
$$

Proof. Fix $\omega=\left[e=\omega_{0}, \omega_{1}, \ldots, \omega_{n}, \ldots\right) \in \partial T$. Let us parametrize the vertices $u$ of $T$ by $(n, k)$, where $n=|u \wedge \omega|$ and $k=|u-u \wedge \omega|$. Fix $n \geq 0$ and restrict attention to those vertices $u$ such that $u \wedge \omega=\omega_{n}$. On this set of vertices $B(u, \omega)$ is a radial function of $u$. Thus, by (3.3), it is determined by the recurrence relation

$$
\frac{q}{q+1} x_{k+1}+\frac{1}{q+1} x_{k-1}-x_{k}=-q^{2 n} q^{-3(n+k)}
$$

and the initial conditions

$$
x_{0}=B\left(\omega_{n}, \omega\right), x_{1}=B\left(\omega_{n}^{+}, \omega\right),
$$

where $\omega_{n}^{+}$is any child of $\omega_{n}$ such that $\omega_{n}^{+} \wedge \omega=\omega_{n}$. 
Let us first consider the case where $n>0$, i.e. $u \wedge \omega \neq e$. A straightforward combinatorial argument gives $x_{0}$ as

$$
\begin{aligned}
x_{0}= & \frac{q}{q-1}\left[\sum_{j=n}^{\infty}\left(\frac{q^{2 j}}{q^{j-n} q^{3 j}}+\sum_{k=1}^{\infty} \frac{q^{2 j}}{q^{j-n+k} q^{3(j+k)}}(q-1) q^{k-1}\right)\right. \\
& \left.+\sum_{j=1}^{n-1}\left(\frac{q^{2 j}}{q^{n-j} q^{3 j}}+\sum_{k=1}^{\infty} \frac{q^{2 j}}{q^{n-j+k} q^{3(j+k)}}(q-1) q^{k-1}\right)+\frac{1}{q^{n}}+\sum_{k=1}^{\infty} \frac{q^{k}}{q^{n+k} q^{3 k}}\right] .
\end{aligned}
$$

The sum reduces to

$$
x_{0}=\left[\frac{q^{4}+q^{2}+1+n\left(q^{4}-1\right)}{(q-1)\left(q^{3}-1\right)}\right] q^{-n} .
$$

We could write down for $x_{1}$ a similar but more complicated expression than the one for $x_{0}$ and sum it in a similar way, but we have a simpler way to get $x_{1}$. We solve the recurrence relation in (3.8) using the value of $x_{0}$ just obtained with $x_{1}$ left in symbolic form. The result is

$$
\begin{aligned}
x_{k}=\{ & x_{1}\left(q^{2}-q^{3}-q^{5}+q^{6}\right)+\left(n q^{1-n}-2 q^{3-n}-(n+1) q^{5-n}\right) \\
& q^{-k}\left[x_{1}\left(-q^{2}+q^{3}+q^{5}-q^{6}\right)-q^{1-n}+q^{6-n}(n+1)-q^{2-n}(n-1)+2 q^{4-n}\right] \\
& \left.+q^{-3 k}\left[q^{3-n}-q^{4-n}\right]\right\} /\left[q(q-1)^{2}\left(q^{3}-1\right)\right] .
\end{aligned}
$$

But we know from (3.4) that $x_{k} \rightarrow 0$ as $k \rightarrow \infty$, so letting $k \rightarrow \infty$ we obtain

$$
x_{1}\left(q^{2}-q^{3}-q^{5}+q^{6}\right)+\left(n q^{1-n}-2 q^{3-n}-(n+1) q^{5-n}\right)=0 .
$$

Solving this for $x_{1}$ and simplifying gives

$$
x_{1}=\left[\frac{q^{2}\left(q^{2}+2\right)+n\left(q^{4}-1\right)}{q\left(q^{3}-1\right)(q-1)}\right] q^{-n} .
$$

Plugging this back into the formula for $x_{k}$, we get

$$
x_{k}=\frac{q^{-n-k}}{(q-1)\left(q^{3}-1\right)}\left[\left(q^{2}+1\right)^{2}+n\left(q^{4}-1\right)-q^{2} q^{-2 k}\right] \text { in case } n>0 .
$$

Consider now the case that $n=0$, i.e. $u \wedge \omega=e$. We use the same method as above to calculate $x_{k}$. The formula for $x_{0}$ is obtained as before using the last sum in (3.1). It turns out to be the same as in (3.10) with $n$ replaced by 0 , except the first sum in (3.10) is taken over all $j \geq 1$ (instead of $j \geq 0$ ) and the sum from $j=1$ to $n-1$ is empty and so is taken to be 0 . The sum is

$$
x_{0}=\frac{q^{2}-q+1}{(q-1)^{2}} .
$$

Solving the equivalence relation for $x_{k}$, letting $k \rightarrow \infty$, equating to 0 and solving for $x_{1}$ gives

$$
x_{1}=\frac{q\left(q^{2}+2\right)}{(q-1)\left(q^{3}-1\right)} \text {. }
$$


Plugging this value back into the recurrence relation and solving gives

$$
x_{k}=\frac{q^{-k}}{(q-1)\left(q^{3}-1\right)}\left[\left(q^{2}+1\right)^{2}-q^{2} q^{-2 k}\right] \text { for } n=0 .
$$

We see that this agrees with formula (3.11) when $n=0$.

Recalling that $|u \wedge \omega|=n,|u-u \wedge \omega|=k$ and $|u|=n+k$, we obtain formula (3.6).

The formula in the theorem makes it easy to calculate the normal derivative of $B \mu(u)$ in the following useful special case.

Corollary 3.1. Let $\mu$ be as in Theorem 3.2. Suppose $\omega \in \partial T$ has the property that for all $\omega^{\prime}$ in the support of $\mu, \omega \wedge \omega^{\prime}$ is the same vertex $v$. Then

$$
\partial_{n} B \mu(\omega)=\frac{\left(q^{2}+1\right)^{2}+\left(q^{4}-1\right)|v|}{(q-1)\left(q^{3}-1\right)}\|\mu\|,
$$

where $\|\mu\|$ is the total mass of $\mu$.

Proof. Let $k_{0} \in \mathbb{N}$ be such that for all integers $k$ greater than $k_{0}, \omega_{k} \wedge \omega^{\prime}=v$ for all $\omega^{\prime}$ in the support of $\mu$. By formula (3.7), we obtain

$$
\partial_{n} B \mu(\omega)=\lim _{k \rightarrow \infty} \frac{\left(q^{2}+1\right)^{2}+\left(q^{4}-1\right)|v|-q^{2} q^{-2\left|\omega_{k}-v\right|}}{(q-1)\left(q^{3}-1\right)}\|\mu\|,
$$

yielding the result.

Fix a vertex $v$. Let $|v|=n$ and $[e, v]=\left[v_{0}, v_{1}, \ldots, v_{n}\right]$ the geodesic from the root to $v$. For each nonnegative integer $j$, define intervals $I_{j}$ and sectors $S_{j}$ by

$$
I_{j}=\left\{\begin{array}{ll}
I_{v_{j}} & \text { if } 0 \leq j \leq n \\
\emptyset & \text { if } j>n
\end{array} \text { and } S_{j}= \begin{cases}S_{v_{j}} & \text { if } 0 \leq j \leq n \\
\emptyset & \text { if } j>n\end{cases}\right.
$$

For each $m=0, \ldots, n$, let $\mu_{m}$ denote unit Lebesgue measure with support in $I_{m}-I_{m+1}$.

The following result follows from (3.7), symmetry, and Corollary 3.1.

Corollary 3.2. Fix a vertex $v$. Using the above notation, the function $B \mu_{m}$ is radial on $S_{j}-S_{j+1}$ and $\partial_{n} B \mu_{m}$ is constant on $I_{j}-I_{j+1}$ for each $j=0, \ldots,|v|$.

\section{Normal derivative of biharmonic Martin integrals of Lebesgue measure on an interval}

In this section, we fix a vertex $v$ of length $n$ and calculate the $(n+1)$ values of $\partial_{n} B \mu_{m}$ guaranteed by Corollary 3.2.

By Corollary 3.1, the values of $\partial_{n} B \mu_{m}$ on $I_{j}-I_{j+1}$ in case $j \neq m$ can be easily determined, while the case $j=m$ is more difficult to treat. In order to handle this case, we first calculate $K_{H} \mu_{m}$. 
Theorem 4.1. For $u \in T$ define the integers $j$ and $k$ by $j=|u \wedge v|$, let $k=|u-u \wedge v|$. Then $K_{H} \mu_{m}(u)$ is given by

$$
K_{H} \mu_{m}(u)= \begin{cases}\left(\frac{q+1}{q}-\frac{1}{q^{1+k}}\right) q^{m} & \text { if } m=j=0 \text { or } m=j=n, \\ \left(\frac{q+1-2 q^{-k}}{q-1}\right) q^{m} & \text { if } 0<m=j<n, \\ q^{2 m-j-k} & \text { if } 0 \leq m<j \leq n, \\ q^{j-k} & \text { if } 0 \leq j<m \leq n .\end{cases}
$$

Proof. Since $K_{H} \mu_{m}$ is harmonic on $T$ and radial on each of the sets $S_{j}-S_{j+1}$, it is a linear combination of 1 and $q^{-k}$ there. Thus its value is determined by the values $K_{H} \mu_{m}\left(v_{j}\right)$ and $K_{H} \mu_{m}\left(v_{j}^{+}\right), j=0, \ldots, n$, where $v_{j}^{+}$is any child of $v_{j}$ in $S_{j}-S_{j+1}$. Using formula (1.8), we obtain the following.

For $m=0=j<n, K_{H} \mu_{m}\left(v_{j}\right)=1, K_{H} \mu_{m}\left(v_{j}^{+}\right)=\frac{1+q}{q}-\frac{1}{q^{2}}$.

For $m=0<j \leq n, K_{H} \mu_{m}\left(v_{j}\right)=q^{-j}, K_{H} \mu_{m}\left(v_{j}^{+}\right)=q^{-j-1}$.

For $0 \leq j<m<n, K_{H} \mu_{m}\left(v_{j}\right)=q^{j}, K_{H} \mu_{m}\left(v_{j}^{+}\right)=q^{j-1}$.

For $0<j=m<n, K_{H} \mu_{m}\left(v_{j}\right)=q^{m}, K_{H} \mu_{m}\left(v_{j}^{+}\right)=q^{m-1}\left(\frac{q-2}{q-1}\right)+\frac{q^{m+1}}{q-1}=$ $(q+2) q^{m-1}$.

For $0<m<j \leq n, K_{H} \mu_{m}\left(v_{j}\right)=q^{2 m-j}, K_{H} \mu_{m}\left(v_{j}^{+}\right)=q^{2 m-(j+1)}$.

For $0 \leq j<m=n, K_{H} \mu_{m}\left(v_{j}\right)=q^{j}, K_{H} \mu_{m}\left(v_{j}^{+}\right)=q^{j-1}$.

For $0<m=n=j, K_{H} \mu_{m}\left(v_{j}\right)=q^{m}, K_{H} \mu_{m}\left(v_{j}^{+}\right)=q^{m-1}\left(\frac{q-1}{q}\right)+q^{m+1}\left(\frac{1}{q}\right)=$ $\left(\frac{q^{2}+q-1}{q^{2}}\right) q^{m}$.

Extending each of these harmonically and combining some of the cases yields the result.

We now return to the calculation of $\partial_{n} B \mu_{m}$.

Theorem 4.2. Let $v \in T$ with $|v|=n$. For $m, j \in\{0, \ldots, n\}$ let $\mu_{m}$ be unit Lebesgue measure with support in $I_{m}-I_{m+1}$ and let $\omega$ be a boundary point of $T$ such that $|\omega \wedge v|=j$. Then

$$
\partial_{n} B \mu_{m}(\omega)=\frac{q^{2}+1}{(q-1)\left(q^{3}-1\right)} \begin{cases}q^{2}+q+2 & \text { if } m=j=0, \\ q^{2}+1+\left(q^{2}-1\right) j & \text { if } j<m \\ q^{2}+1+\left(q^{2}-1\right) m & \text { if } m<j, \\ \frac{q^{3}+2 q-1}{q-1}+\left(q^{2}-1\right) m & \text { if } 0<m=j<n, \\ q^{2}+q+2+\left(q^{2}-1\right) n & \text { if } m=j=n .\end{cases}
$$

Proof. Of the five equalities in the theorem the second and third follow easily from Corollary 3.1. So assume for the rest of the proof that $m=j$. We consider separately the three cases $0<m<n, m=n$ and $m=0$.

Consider first the case $0<m<n$. The function $B \mu_{m}(u)$ restricted to $S_{m}-$ $S_{m+1}$ is radial with respect to the distance from $u$ to $u \wedge v_{m}$. Denote this distance 
by $k$ and the value of the function by $x_{k}$. By (3.2) we have

$$
\Delta_{H}\left(B \mu_{m}\right)(u)=-\frac{K_{H} \mu_{m}(u)}{q^{2|u|}} .
$$

Thus by Theorem 4.1 we deduce

$$
\frac{q}{q+1} x_{k+1}+\frac{1}{q+1} x_{k-1}-x_{k}=-\left(\frac{q+1-2 q^{-k}}{q-1}\right) q^{-m-2 k},
$$

so the values of $x_{k}$ are determined by this recurrence relation and the values

$$
x_{0}=B \mu_{m}\left(v_{m}\right) \text { and } x_{1}=B \mu_{m}\left(v_{m}^{+}\right)
$$

where $v_{m}^{+}$is defined as in the proof of Theorem 4.1. These are each calculated easily using (3.7). We get

$$
x_{0}=\frac{q^{-m}}{(q-1)\left(q^{3}-1\right)}\left[\left(q^{2}+1\right)^{2}+\left(q^{4}-1\right) m-q^{2}\right]
$$

and

$$
x_{1}=\frac{q^{-(m+1)}}{(q-1)\left(q^{3}-1\right)}\left[\left(q^{2}+q+3\right) q^{2}+\left(q^{4}-1\right) m\right] .
$$

Solving recurrence relation (4.2) with these values of $x_{0}$ and $x_{1}$ gives

$$
\begin{gathered}
x_{k}=\frac{q^{-(m+k)}}{(q-1)^{2}\left(q^{3}-1\right)}\left[\left(q^{2}+1\right)\left(q^{3}+2 q-1\right)+\left(q^{2}+1\right)(q+1)(q-1)^{2} m+\right. \\
\left.-q^{-k} q(q+1)\left(q^{2}+q+1\right)+2 q^{-2 k} q^{2}\right]
\end{gathered}
$$

Then, for $0<m<n$ and $\omega \in I_{m}-I_{m+1}$, multiplying by $q^{|u|}=q^{m+k}$ and letting $k \rightarrow \infty$ yields

$$
\partial_{n} B \mu_{m}(\omega)=\frac{\left(q^{2}+1\right)\left[q^{3}+2 q-1+m(q+1)(q-1)^{2}\right]}{\left(q^{3}-1\right)(q-1)^{2}},
$$

in agreement with the formula to be proved.

Consider next the case $m=n$. Using notation as above and a similar technique, we obtain that the sequence $\left\{x_{k}\right\}$ satisfies the recurrence relation

$$
\frac{q}{q+1} x_{k+1}+\frac{1}{q+1} x_{k-1}-x_{k}=-\left(\frac{q+1}{q}-\frac{1}{q^{1+k}}\right) q^{-n-2 k},
$$

and the initial conditions

$$
\begin{aligned}
& x_{0}=B \mu_{n}\left(v_{n}\right)=\frac{q^{-n}}{(q-1)\left(q^{3}-1\right)}\left[\left(q^{2}+1\right)^{2}+\left(q^{4}-1\right) n-q^{2}\right], \\
& x_{1}=\frac{q^{-n-1}}{(q-1)\left(q^{3}-1\right)}\left[n\left(q^{4}-1\right)+q\left(q^{3}+q^{2}+2 q-1\right)\right] .
\end{aligned}
$$


The solution of the recurrence relation is given by

$$
\begin{array}{r}
x_{k}=q^{-(n+k)}\left[\frac{n\left(q^{4}-1\right)}{(q-1)\left(q^{3}-1\right)}+\frac{\left(q^{2}+q+2\right)\left(q^{2}+1\right)}{(q-1)\left(q^{3}-1\right)}\right. \\
\left.-q^{-k} \frac{(q+1)}{(q-1)^{2}}+q^{-2 k} \frac{q}{(q-1)\left(q^{3}-1\right)}\right]
\end{array}
$$

and multiplying by $q^{n+k}$ and letting $k \rightarrow \infty$ gives for $\omega \in I_{n}-I_{n+1}$

$$
\partial_{n} B \mu_{n}(\omega)=\frac{\left(q^{2}+1\right)\left[q^{2}+q+2+n\left(q^{2}-1\right)\right]}{(q-1)\left(q^{3}-1\right)} .
$$

For the case of $m=0$, the results for each of $x_{0}, x_{1}, x_{k}$ and $\partial_{n} B \mu_{0}(\omega)$ are the same as the case of $m=n$ with $n$ replaced by 0 .

\section{Existence of the flat clamped biharmonic Green function}

In this section our aim is to prove the existence of $\Gamma_{C}(u, v)$, the flat clamped biharmonic Green function. We will accomplish this by exploiting the simplysupported Green function $\Gamma_{S}$ and reducing the problem to proving that a certain matrix needed for the construction is non-singular. We need some preliminary results.

Recall that a Toeplitz matrix is a matrix with constant diagonal entries from left to right. Given $a, b \in \mathbb{R}$, we shall call symmetric Toeplitz matrix with parameters $a$ and $b$ the Toeplitz matrix whose entries on the main diagonal are equal to $a$, whose entries adjacent to the main diagonal are equal to $b$, and all other entries are 0 .

Lemma 5.1. Let $a, b \in \mathbb{R}$ and let $T_{n}$ be the determinant of the symmetric Toeplitz matrix of order $n$ with parameters $a$ and $b$. Then $T_{0}=1$ and

$$
T_{n}=a T_{n-1}-b^{2} T_{n-2}, \text { for all } n \in \mathbb{N}, n \geq 2 \text {. }
$$

Proof. Expanding $T_{n}$ across the first row we obtain

$$
T_{n}=a T_{n-1}-b\left|\begin{array}{ccccc}
b & b & 0 & \cdots & 0 \\
0 & a & b & \ddots & 0 \\
\vdots & b & \ddots & \ddots & 0 \\
\vdots & \vdots & \ddots & \ddots & b \\
0 & 0 & \cdots & b & a
\end{array}\right|=a T_{n-1}-b^{2} T_{n-2} .
$$

Lemma 5.2. Let $T_{n}$ be the determinant of the symmetric Toeplitz matrix $A_{n}$ of order $n$ with parameters $a$ and $b$. Let

$$
B_{n}=\left[\begin{array}{cc}
A_{n-1} & \mathbf{v}^{T} \\
\mathbf{v} & a+c
\end{array}\right],
$$


where $\mathbf{v}=\left[\begin{array}{llll}0 & \cdots & 0 & b\end{array}\right], c$ is a constant, and the superscript ${ }^{T}$ denotes the transpose operator. Then $\operatorname{det}\left(B_{n}\right)=T_{n}+c T_{n-1}$, for all $n \geq 2$.

Proof. For $n \geq 2$, by expanding the determinant of $B_{n}$ across the bottom row and using Lemma 5.1, we obtain

$$
\operatorname{det}\left(B_{n}\right)=(a+c) T_{n-1}-b^{2} T_{n-2}=T_{n}+c T_{n-1} .
$$

Lemma 5.3. For each $n \in \mathbb{N}$, let $D_{n+1}$ be the symmetric matrix of order $n+1$ defined by

$$
\left[\begin{array}{cccc}
\left(q^{2}+q+2\right)(q-1) & -(q+1)(q-1) & \mathbf{0} & 0 \\
-(q+1)(q-1) & q^{2}(q+1) & \mathbf{w} & \mathbf{0} \\
\mathbf{0} & \mathbf{w}^{T} & A_{n-2} & \mathbf{v}^{T} \\
0 & \mathbf{0} & \mathbf{v} & q^{2}(q+1)
\end{array}\right]
$$

where $\mathbf{w}=\left[\begin{array}{llll}-q(q+1) & 0 & \cdots & 0\end{array}\right], \mathbf{v}=\left[\begin{array}{llll}0 & \cdots & 0 & -q(q+1)\end{array}\right]$, and $A_{n-2}$ is the symmetric Toeplitz matrix of order $n-2$ with parameters $(q+1)\left(q^{2}+1\right)$ and $-q(q+1)$. Then $D_{n+1}$ is non-singular.

Proof. Observe that

$$
\operatorname{det}\left(D_{n+1}\right)=(q-1)(q+1)^{n} \operatorname{det}\left(E_{n+1}\right),
$$

where

$$
E_{n+1}=\left[\begin{array}{cccc}
q^{2}+q+2 & -(q+1) & \mathbf{0} & 0 \\
-(q-1) & q^{2} & \mathbf{w}^{\prime} & \mathbf{0} \\
\mathbf{0} & \mathbf{w}^{\prime T} & A_{n-2}^{\prime} & \mathbf{v}^{\prime T} \\
0 & \mathbf{0} & \mathbf{v}^{\prime} & q^{2}
\end{array}\right]
$$

with $\mathbf{w}^{\prime}=\left[\begin{array}{llll}-q & 0 & \cdots & 0\end{array}\right], \mathbf{v}^{\prime}=\left[\begin{array}{llll}0 & \cdots & 0 & -q\end{array}\right]$, and $A_{n-2}^{\prime}$ is the symmetric Toeplitz matrix of order $n-2$ with parameters $q^{2}+1$ and $-q$. By a straightforward calculation and the use of Lemma 5.2 with $a=q^{2}+1, b=-q, c=-1$, and $T_{n}=\operatorname{det}\left(A_{n}^{\prime}\right)$, we obtain

$$
\begin{aligned}
\operatorname{det}\left(E_{n+1}\right)= & \left(q^{2}+q+2\right)\left[q^{2}\left(T_{n-1}-T_{n-2}\right)-q^{2}\left(T_{n-2}-T_{n-3}\right)\right] \\
& -\left(q^{2}-1\right)\left(T_{n-1}-T_{n-2}\right) \\
= & \left(q^{2}+q+2\right) q^{2}\left(T_{n-1}-2 T_{n-2}+T_{n-3}\right)-\left(q^{2}-1\right)\left(T_{n-1}-T_{n-2}\right) .
\end{aligned}
$$

By Lemma 5.1, $T_{n}$ satisfies the second order linear recurrence relation

$$
T_{n+1}=\left(q^{2}+1\right) T_{n}-q^{2} T_{n-1}
$$

for $n \geq 2$, with the initial conditions $T_{1}=q^{2}+1$ and $T_{2}=\left(q^{2}+1\right)^{2}-q^{2}$. The roots of the characteristic equation $r^{2}-\left(q^{2}+1\right) r+q^{2}=0$ are 1 and $q^{2}$, so $T_{n}=\alpha+\beta q^{2 n}$ for $n \geq 1$. From the initial conditions, we obtain $\alpha=-\frac{1}{q^{2}-1}$ and $\beta=\frac{q^{2}}{q^{2}-1}$. We deduce 
$T_{n}=\frac{1}{q^{2}-1}\left(q^{2(n+1)}-1\right)$ for $n \geq 1$. Thus, $T_{n-1}-2 T_{n-2}+T_{n-3}=\left(q^{2}-1\right) q^{2(n-2)}$, and $T_{n-1}-T_{n-2}=q^{2(n-1)}$. Hence using (5.2), we obtain

$\operatorname{det}\left(E_{n+1}\right)=\left(q^{2}+q+2\right)\left(q^{2}-1\right) q^{2(n-1)}-\left(q^{2}-1\right) q^{2(n-1)}=\left(q^{2}-1\right)\left(q^{2}+q+1\right) q^{2(n-1)}$.

From (5.1), we conclude $\operatorname{det}\left(D_{n+1}\right)=(q-1)^{2}(q+1)^{n+1}\left(q^{2}+q+1\right) q^{2(n-1)}$. Therefore $D_{n+1}$ is non-singular for all $n \in \mathbb{N}$.

We are now ready to prove the existence of $\Gamma_{C}$.

Theorem 5.1. There exists a function $\Gamma_{C}$ on $\bar{T} \times T$ satisfying the conditions of (1.13). In addition, for each fixed vertex $v, \Gamma_{C}(u, v)$ is radial on each set $S_{j}-S_{j+1}$ (notation as in (3.13)) and can be written in the form

$$
\Gamma_{C}(u, v)=\frac{x_{C}(u \wedge v, v)}{q^{2|u-u \wedge v|}}+\frac{y_{C}(u \wedge v, v)}{q^{3|u-u \wedge v|}}
$$

for appropriate functions $x_{C}$ and $y_{C}$.

Proof. We proceed by showing that there exists a function $\Phi(u, v)$ which, for each fixed $v$, is of the form $B \mu(u)$ for some measure $\mu$ which is a linear combination of unit Lebesgue measures with supports on $I_{j}-I_{j+1}, j=0, \ldots|v|$, and for every $\omega \in \partial T$

$$
\partial_{n} \Phi(\omega, v)=\partial_{n} \Gamma_{S}(\omega, v) .
$$

Then by Theorem 3.1, if $\Gamma_{C}$ is defined by

$$
\Gamma_{C}(u, v)=\Gamma_{S}(u, v)-\Phi(u, v),
$$

it will have the properties in (1.13).

Fix a vertex $v$ and let $n=|v|$. For each $\omega \in \partial T$, by formulas (1.11) and (2.2), and recalling from $(1.12)$ that $\Gamma_{S}(\omega, v)=0$, we obtain

$$
\begin{aligned}
\partial_{n} \Gamma_{S}(\omega, v) & =\lim _{m \rightarrow \infty} q^{m}\left(\Gamma_{S}\left(\omega_{m}, v\right)-\Gamma_{S}(\omega, v)\right) \\
& =\lim _{m \rightarrow \infty} q^{m}\left[\frac{x_{S}\left(\omega_{m} \wedge v, v\right)}{q^{\left.\mid \omega_{m}-\omega_{m} \wedge v\right)}}+\frac{y_{S}\left(\omega_{m} \wedge v, v\right)}{q^{\left.3 \mid \omega_{m}-\omega_{m} \wedge v\right)}}\right] \\
& =q^{|\omega \wedge v|} x_{S}(\omega \wedge v, v) .
\end{aligned}
$$

Thus, in order to prove the existence of such a function $\Phi(u, v)$ it suffices for us to prove that for each $(|v|+1)$-tuple of real numbers $\left(r_{0}, r_{1}, \ldots, r_{|v|}\right)$ there exists a flat biharmonic function on $T$ written as a biharmonic Martin integral of a finite linear combination of unit Lebesgue measures on $I_{j}-I_{j+1}, j=0, \ldots,|v|$, such that for each $j \in\{0, \ldots,|v|\}$ and $\omega \in \partial T$ with $|\omega \wedge v|=j$, its normal derivative equals $r_{j}$.

Consider the following matrix $M$ which contains a fixed multiple of all of the values of the normal derivative of $B \mu_{m}$, namely

$$
M_{m, j}=\frac{(q-1)\left(q^{3}-1\right)}{q^{2}+1} \partial_{n} B \mu_{m}(\omega), \text { where }|\omega \wedge v|=j .
$$

This defines an $(n+1) \times(n+1)$-matrix in which the indices $m$ and $j$ run from 0 to $n$. In order to show the existence of the above function $\Phi$ it suffices to show 
that $M$ is nonsingular. Referring to (4.1) we note that $M$ is a symmetric matrix with the property that in each column all of the entries below the diagonal are the same.

Let us perform the following sequence of row and column operations on $M$ (where we call the rows $R_{0}, R_{1}, \ldots, R_{n}$ and the columns $C_{0}, C_{1}, \ldots, C_{n}$ ):

$$
\begin{array}{cccc}
R_{n} \rightarrow R_{n}-R_{n-1}, & R_{n-1} \rightarrow R_{n-1}-R_{n-2}, & \ldots, & R_{1} \rightarrow R_{1}-R_{0}, \\
C_{n} \rightarrow C_{n}-C_{n-1}, & C_{n-1} \rightarrow C_{n-1}-C_{n-2}, & \ldots, & C_{1} \rightarrow C_{1}-C_{0} .
\end{array}
$$

Multiply each of the elements of the resulting matrix by $q-1$. We then obtain a symmetric matrix whose nonzero entries all lie in the main diagonal and in the two diagonals adjacent to it. Moreover, the main diagonal entries are

$$
\left(q^{2}+q+2\right)(q-1), \quad(q+1) q^{2}, \quad \underbrace{(q+1)\left(q^{2}+1\right)}_{n-2 \text { copies }}, \quad(q+1) q^{2}
$$

and the entries in the adjacent diagonals are

$$
-\left(q^{2}-1\right), \quad \underbrace{-q(q+1)}_{n-1 \text { copies }} .
$$

Observe that this is precisely the non-singular matrix $D_{n+1}$ of Lemma 5.3. This completes the proof of the existence of a function $\Gamma_{C}(u, v)$ satisfying (1.13).

The radiality assertion follows from (5.4), Theorem 2.2 and Corollary 3.2, and formula (5.3) then follows from Proposition 1.1.

\section{Uniqueness of the flat clamped biharmonic Green function}

In this section, we show that the flat clamped biharmonic Green function is unique. The proof, motivated by the proof in [14, Section 1.6] of the analogous result on the unit disk in the complex plane, makes use of a Green formula together with the existence (established in Section 5) of at least one clamped biharmonic Green function having a slightly stronger property than is evident from the definition (namely formula (5.3)). Let us denote by $\Gamma_{C}$ the function defined in Theorem 5.1.

Given a function $g$ on $T$ and a non-negative integer $N$, define

$$
M_{N} g(u)=\max _{|u-w| \leq N}|g(w)| .
$$

For functions $g, h$ on $T$ and for an integer $k$, when we write $h=q^{k|u|} \mathcal{O}(g)$, we mean that for some positive constants $C$ and $N$ we have

$$
|h(u)| \leq C q^{k|u|} M_{N} g(u),
$$

for every $u \in T$.

In the proof we shall make use of both kinds of normal derivative defined in (1.9) and (1.11).

Lemma 6.1. For any function $g$ on $T, \frac{\partial g}{\partial n}=\mathcal{O}(g)$ and $\Delta_{E} g(u)=q^{2|u|} \mathcal{O}(g(u))$. 
Proof. We have $\left|g(u)-g\left(u^{-}\right)\right| \leq|g(u)|+\left|g\left(u^{-}\right)\right| \leq 2 M_{1} g(u)$. Since $\Delta_{H} g(u)=$ $\sum_{v \sim u}(g(v)-g(u)) /(q+1)$, it follows that $\left|\Delta_{H} g(u)\right| \leq 2 M_{1} g(u)$ so $\left|\Delta_{E} g(u)\right| \leq$ $q^{2|u|} M_{1} g(u)$.

The proof of the next lemma follows from the fact that there are less than $2 q^{n}$ vertices of length $n$ and the compactness of $T \cup \partial T$ with respect to the flat metric.

Lemma 6.2. If $h$ is a function on $T$ such that $\lim _{u \rightarrow \partial T} q^{|u|} h(u)=0$ then

$$
\lim _{N \rightarrow \infty} \sum_{|u|=N} h(u)=0 .
$$

Lemma 6.3. Let $w$ be a fixed vertex. Then

1. $\Gamma_{C}(u, w)=\mathcal{O}\left(q^{-2|u|}\right)$,

2. $\Delta_{E} \Gamma_{C}(u, w)=\mathcal{O}(1)$,

for all $v \in T$.

Proof. The first follows immediately from (5.3). The second follows from the same formula upon taking the flat Laplacian in the $u$ variable at points a distance at least 1 from the union of the points of the geodesic joining $v$ to $e$.

In [12, Theorem 4.1], we gave a formulation of Green's theorem on trees, which we state below for the special case of a homogeneous tree $T$ of degree $q+1$ and for a ball centered at $e$.

Theorem 6.1 ([12]). For $N \in \mathbb{N}$, let $B_{N}=\{u \in T:|u| \leq N\}$, and let $f, g$ be functions on $T$. Then

$$
\sum_{|u|<N} \Delta_{H} f(u) g(u)+\mathbf{D}_{B_{N}}(f, g)=\frac{1}{q+1} \sum_{|u|=N} \frac{\partial f}{\partial n}(u) g\left(u^{-}\right),
$$

where $\mathbf{D}_{B_{N}}$ denotes the Dirichlet sum defined by

$$
\mathbf{D}_{B_{N}}(f, g)=\frac{1}{q+1} \sum_{u, u^{-},|u|<N}\left(f(u)-f\left(u^{-}\right)\right)\left(g(u)-g\left(u^{-}\right)\right) .
$$

Using the symmetry of the Dirichlet sum and recalling the relation between the hyperbolic and the flat Laplacian, we obtain the following result.

Theorem 6.2. (Green's formula) Given functions $f, g$ on $T$, and a nonnegative integer $N$,

$$
\sum_{|u|<N} q^{-2|u|}\left[\Delta_{E} f(u) g(u)-\Delta_{E} g(u) f(u)\right]=\frac{1}{q+1} \sum_{|u|=N}\left[\frac{\partial f}{\partial n}(u) g\left(u^{-}\right)-\frac{\partial g}{\partial n}(u) f\left(u^{-}\right)\right] .
$$

We are now ready to prove our main result.

Theorem 6.3. The clamped biharmonic Green function is unique. 
Proof. Assume $\Gamma_{1}$ and $\Gamma_{2}$ are clamped biharmonic Green functions. Fix any vertex $w$ in $T$ and let $f=\Gamma_{1}(\cdot, w)-\Gamma_{2}(\cdot, w)$. We must show that $f$ is identically 0 .

The function $f$ is flat biharmonic on $T$ and has the property that both $f(u) \rightarrow 0$ as $|u| \rightarrow \infty$, and for every $\omega$ in the boundary of $T, \partial_{n} f(\omega)=0$. By the compactness of $T \cup \partial T$, we deduce that $f(u) q^{|u|} \rightarrow 0$ as $|u| \rightarrow \infty$.

Letting $N \in \mathbb{N}, n>|w|$, and applying Green's formula to $B_{N}$ and the functions $\Delta_{E} f$ and $\Gamma_{C}(\cdot, w)$, we obtain

$$
\begin{aligned}
-\sum_{|u|<N} q^{-2|u|} \Delta_{E} \Gamma_{C}(u, w) \Delta_{E} f(u) \\
\quad=\frac{1}{q+1} \sum_{|u|=N}\left[\frac{\partial \Delta_{E} f}{\partial n}(u) \Gamma_{C}\left(u^{-}, w\right)-\frac{\partial \Gamma_{C}}{\partial n}(u, w) \Delta_{E} f\left(u^{-}\right)\right] .
\end{aligned}
$$

We next apply Green's formula to the functions $\Delta_{E} \Gamma_{C}(\cdot, w)$ and $f$. Since $\Delta_{E}^{2} \Gamma_{C}(\cdot, w)=\delta_{w}$, we get

$$
\begin{aligned}
q^{-2|w|} f(w) & -\sum_{|u|<N} q^{-2|u|} \Delta_{E} f(u) \Delta_{E} \Gamma_{C}(u, w) \\
& =\frac{1}{q+1} \sum_{|u|=N}\left[\frac{\partial \Delta_{E} \Gamma_{C}}{\partial n}(u, w) f\left(u^{-}\right)-\frac{\partial f}{\partial n}(u) \Delta_{E} \Gamma_{C}\left(u^{-}, w\right)\right] .
\end{aligned}
$$

Combining (6.1) and (6.2), we obtain

$$
q^{-2|w|} f(w)=\frac{1}{q+1}(I-I I-I I I+I V),
$$

where

$$
\begin{gathered}
I=\sum_{|u|=N} \frac{\partial \Delta_{E} \Gamma_{C}}{\partial n}(u, w) f\left(u^{-}\right) \\
I I=\sum_{|u|=N} \frac{\partial f}{\partial n}(u) \Delta_{E} \Gamma_{C}\left(u^{-}, w\right) \\
I I I=\sum_{|u|=N} \frac{\partial \Delta_{E} f}{\partial n}(u) \Gamma_{C}\left(u^{-}, w\right) \\
I V=\sum_{|u|=N} \frac{\partial \Gamma_{C}}{\partial n}(u, w) \Delta_{E} f\left(u^{-}\right) .
\end{gathered}
$$

To complete the proof, it suffices to show that each of the terms $I, I I, I I I$, and $I V$ goes to 0 as $N \rightarrow \infty$.

By Lemmas 6.3(2) and 6.1, $\frac{\partial \Delta_{E} \Gamma_{C}}{\partial n}(\cdot, w)=\mathcal{O}(1)$. Since $q^{|u|} f(u) \rightarrow 0$ as $|u| \rightarrow$ $\infty$, using Lemma 6.2, we deduce that $I \rightarrow 0$ as $N \rightarrow \infty$. Similarly, $\frac{\partial f}{\partial n}=\mathcal{O}(f)$ and $\Delta_{E} \Gamma_{C}(\cdot, w)=\mathcal{O}(1)$, so that $I I \rightarrow 0$ as $N \rightarrow \infty$. Moreover, by Lemmas 6.1 and 
$6.3(1)$, we obtain

$$
\frac{\partial \Delta_{E} f}{\partial n}(u) \Gamma_{C}\left(u^{-}, w\right)=q^{2|u|} \mathcal{O}(f(u)) \mathcal{O}\left(q^{-2|u|}\right)=\mathcal{O}(f(u)) .
$$

Consequently, since the sum in $I I I$ has $(q+1) q^{N-1}$ terms in it,

$$
I I I=q^{N} \max _{|u|=N}|f(u)| \mathcal{O}(1) \rightarrow 0,
$$

as $N \rightarrow \infty$. Finally,

$$
\frac{\partial \Gamma_{C}}{\partial n}(u, w) \Delta_{E} f\left(u^{-}\right)=\mathcal{O}\left(q^{-2|u|}\right) q^{2 \mid u^{-}} \mid \mathcal{O}\left(f\left(u^{-}\right)\right)=\mathcal{O}\left(f\left(u^{-}\right)\right),
$$

so, by a similar argument as for $I I I, I V \rightarrow 0$ as $N \rightarrow \infty$, completing the proof.

\section{References}

[1] A. Abkar, On the integral representation of superbiharmonic functions (English summary), Czechoslovak Math. J. 57 (132) (no. 3) (2007), 877-883.

[2] A. Abkar, A new biharmonic kernel for the upper half plane (English summary), J. Korean Math. Soc. 43 (no. 6) (2006), 1169-1181.

[3] A. Abkar and H. Hedenmalm, A Riesz representation formula for super-biharmonic functions, Ann. Acad. Sci. Fenn. Math. 26 (no. 2) (2001), 305-324.

[4] L.E. Andersson, T. Elfving and G.H. Golub, Solution of biharmonic equations with application to radar imaging, J. Comput. Appl. Math. 94 (1998), 153-180.

[5] C.A. Berenstein, E. Casadio Tarabusi, J.M. Cohen and M.A. Picardello, Integral geometry on trees, Amer. J. Math. 113 (no. 3) (1991), 441-470.

[6] P. Cartier, Fonctions harmoniques sur un arbre, Symposia Mathematica vol. 9, Academic Press, London, 1972, 203-270.

[7] E. Casadio Tarabusi, J.M. Cohen and F. Colonna, Range of the horocyclic Radon transform on trees, Ann. Inst. Fourier (Grenoble) 50 (no. 1) (2000), 211-234.

[8] E. Casadio Tarabusi, J.M. Cohen, A. Korányi and A.M. Picardello, Converse mean value theorems on trees and symmetric spaces (English summary), J. Lie Theory 8 (no. 2) (1998), 229-254.

[9] E. Casadio Tarabusi, J. M. Cohen and A. M. Picardello, Range of the X-ray transform on trees, Adv. Math. 109 (no. 2) (1994), 153-167.

[10] J.M. Cohen and F. Colonna, The functional analysis of the X-ray transform on trees (English summary), Adv. in Appl. Math. 14 (no. 1) (1993), 123-138.

[11] J.M. Cohen, F. Colonna, K. Gowrisankaran and D. Singman, Polyharmonic functions on trees, Amer. J. Math. 124 (no. 5) (2002), 999-1043.

[12] J.M. Cohen, F. Colonna and D. Singman, A global Riesz decomposition theorem on trees without positive potentials, (English summary) J. London Math. Soc. (2) 75 (2007), 1-17.

[13] J.M. Cohen, M. Pagliacci and M.A. Picardello, Radial heat diffusion from the root of a homogeneous tree and the combinatorics of paths, (English summary) Boll. Unione Mat. Ital. (9) 1 (2008), 619-628. 
[14] P. Duren and A. Schuster, Bergman Spaces, Mathematical Surveys and Monographs 100, American Mathematical Society, Providence, RI, 2004.

[15] K. Dalrymple, R.S. Strichartz and J.P. Vinson, Fractal differential equations on the Sierpinski gasket (English summary), J. Fourier Anal. Appl. 5 (no. 2-3) (1999), 203284.

[16] A. Figà-Talamanca and M. A. Picardello, Harmonic Analysis on Free Groups, Lecture Notes in Pure and Applied Mathematics 87, Marcel Dekker, Inc., New York, 1983.

[17] K. Gowrisankaran and D. Singman, Tangential limits of potentials on homogeneous trees, Potential Anal. 18 (no. 1) (2003), 79-96.

[18] J. Kigami, A harmonic calculus on the Sierpiński spaces, Japan J. Appl. Math. 6 (no. 2) (1989), 259-290.

[19] A. Korányi and M. A. Picardello, Boundary behaviour of eigenfunctions of the Laplace operator on trees, Ann. Scuola Norm. Sup. Pisa Cl. Sci. (4) 13 (1986), 389399.

[20] S.A. Lurie and V.V. Vasiliev, The Biharmonic Problem in the Theory of Elasticity, Gordon and Breach Publishers, Luxembourg, 1995.

[21] J. Ralston and L. Sario, A relation between biharmonic Green's functions of simply supported and clamped bodies, Nagoya Math. J. 61 (1976), 59-71.

[22] W. Rudin, Function Theory in the Unit Ball of $\mathbb{C}^{n}$, Fundamental Principles of Mathematical Science 241, Springer-Verlag, New York-Berlin, 1980.

[23] L. Sario, M. Nakai, C. Wang and L.O. Chung, Classification Theory of Riemannian Manifolds. Harmonic, Quasiharmonic and Biharmonic Functions, Lect. Notes in Math. 605, Springer-Verlag, Berlin-New York, 1977.

[24] M.H. Taibleson Hardy spaces of harmonic functions on homogeneous isotropic trees, Math. Nachr. 133 (1987), 273-288.

Joel M. Cohen

University of Maryland

College Park, Maryland, 20742

U.S.A.

e-mail: jmc@math.umd.edu

Flavia Colonna and David Singman

George Mason University

Fairfax, Virginia, 22030

U.S.A.

e-mail: fcolonna@gmu.edu

dsingman@gmu.edu

Received: June 20, 2008.

Revised: March 20, 2009.

Accepted: March 26, 2009. 